\title{
Synthesis and Characterization of Bimetallic Ru-Re Catalysts Supported on Oxide Carriers
}

\author{
Szymon Smykała1, Katarzyna Adamska², Mirosława Pawlyta ${ }^{1}$ \\ ${ }^{1}$ Silesian University of Technology \\ Konarskiego 18a, 44-100 Gliwice, Poland \\ szymon.smykala@polsl.pl; miroslawa.pawlyta@polsl.pl \\ ${ }^{2}$ Institute of Low Temperature and Structure Research \\ Okólna 2, 50-422 Wrocław, Poland \\ k.adamska@intibs.pl
}

\section{Extended Abstract}

To be effective, synthesis of catalytic systems, consisting of metallic nanoparticles supported on oxide carriers has to be repeatable, efficient and at the same time, needs to produce samples of regular dispersion of active phase and precise elemental composition. With this, we present a relatively simple technique of synthesising bimetallic nanoparticles deposited on oxide carriers using microwave-assisted polyol approach. Morphology, microstructure and elemental composition of obtained catalysts were explored by transmission electron microscopy. Bimetallic Ru-Re system already proven to be effective in catalytic oxidation of short-chain hydrocarbons and are currently being tested in a soot combustion reaction. Introducing rhenium into ruthenium lattice increases catalysts dispersion and increases it's stability [1].

Catalysts preparation started with dissolving appropriate amounts of precursors $\left(\mathrm{RuCl}_{3}\right.$ and $\left.\mathrm{NH}_{4} \mathrm{ReO}_{4}\right)$ in ethylene glycol, then stirring overnight. After the dissolution of precursors, oxide powder was added to the solution. Oxide carriers we have chose included $\gamma-\mathrm{Al}_{2} \mathrm{O}_{3}$ and $\mathrm{TiO}_{2}$ in rutile and anatase phase. After further stirring, the mixture was poured into a Teflon tube which was placed inside a microwave reactor. Inside the reactor, in the presence of ethylene glycol and thanks to elevated temperature and pressure, reduction of ruthenium and rhenium ions occured. During this process, metallic nanoparticles are formed and, under the influence of microwaves, pushed inside the pores of the support. The obtained slurry was decanted and repeatedly washed with $\mathrm{NaNO}_{3}$ solution. Before the examination, all samples were treated with hydrogen for 3 hours under $400{ }^{\circ} \mathrm{C}$. To examine catalysts structure evolution under an oxidizing atmosphere, we tested one sample after heating to $500{ }^{\circ} \mathrm{C}$ for 1 hour in the atmospheric air. Theoretical elemental compositions of catalysts were $2 \% \mathrm{Ru}-0,8 \% \mathrm{Re}$ and $5 \% \mathrm{Ru}-$ $2 \%$ Re.

Catalysts characterisation was carried out using S/TEM TITAN 80-300 microscope equipped with EDAX EDS spectrometer. STEM images were collected with a $24.5-\mathrm{mrad}$ probe semi-angle and recorded by HAADF detector at $47-$ $200 \mathrm{mrad}$ range.

EDS analysis confirmed that synthesised catalysts compositions were in accordance with calculated values. In the case of the sample heated in air, no rhenium could be detected, which supports the concept of Re redispersion in elevated temperatures [2]. Z-contrast imaging allowed for unveiling catalysts morphology. We were able to tell how nanoparticles microstructure depends on which oxide support was chosen. Particles deposited on $\gamma-\mathrm{Al}_{2} \mathrm{O}_{3}$ could be characterised with spherical shapes without any tendency for agglomeration. On the other hand, such trends could be observed in catalysts supported on rutile, where nanoparticles formed arrays consisting of several smaller crystallites. This phenomenon was less apparent for anatase-supported samples, although increasing metal loading seems to promote this kind of behaviour. Sample heated in the atmospheric air had dramatically different morphology than reduced ones. It consisted of large $(\sim 50 \mathrm{~nm})$ crystallites, which suggest, that the catalyst undergoes sintering in elevated temperatures under oxidising atmosphere.

\section{References}

[1] K. Baranoska and J. Okal, "Bimetallic Ru-Re/ $\gamma$-Al2O3 catalysts for the catalytic combustion of propane: Effect of the Re addition," Applied Catalysis A: General, vol. 499, pp. 158-167, 2015.

[2] J. Okal, L. Krajczyk and M. Drozd, "Oxidation and Redispersion of a $\mathrm{Re} / \gamma-\mathrm{Al}_{2} \mathrm{O}_{3}$ Catalyst," Journal of Catlaysis, vol 188, pp. 140-153, 1999. 